\title{
Proposta de um Dispositivo Não Invasivo para Monitoramento do Ambiente do Sono
}

\author{
Vitor S. Campos ${ }^{1}$, Josué A. S. Oliveira ${ }^{1}$, Fábio M. F. Lobato ${ }^{1}$, Josivan R. Reis ${ }^{1}$, \\ Antonio F. L. Jacob Jr ${ }^{2}$, Marcelino S. da Silva ${ }^{3}$, Roberto P. Nascimento ${ }^{1}$ \\ ${ }^{1}$ Universidade Federal do Oeste do Pará (UFOPA) - Santarém, PA - Brasil \\ ${ }^{2}$ Universidade do Estado do Maranhão - São Luís, MA - Brasil \\ ${ }^{3}$ Universidade Federal do Pará (UFPA) - Belém, PA - Brasil \\ \{vituca.9, josuefan56\}@gmail.com \\ \{fabio.lobato, josivan.reis, roberto.nascimento\}@ufopa.edu.br \\ antoniojunior@professor.uema.br, Marcelino@ufpa.br
}

\begin{abstract}
Sleep disorders are problems that affect a significant portion of society and their diagnosis is usually conditioned by the use of the laboratory polysomnography method. However, this is a costly, inconvenient and cumbersome technique to the patient during the screening process. In this sense, the present article presents a low-cost developmental device for monitoring in-situ and noninvasive sleep, designed under the Design Science Research Methodology, which collects the variables of the rest environment, since these aspects have crucial influence on the quality of sleep.
\end{abstract}

Resumo. Os distúrbios no sono são problemas que afetam uma parcela significativa da sociedade e seu diagnóstico normalmente está condicionado a utilização do método de polissonografia laboratorial. No entanto, essa é uma técnica dispendiosa, inconveniente e incômoda ao paciente durante o processo de triagem. Nesse sentido, o presente artigo apresenta um dispositivo em desenvolvimento de baixo custo, para monitoramento do sono in loco e não invasivo, concebido sob o Design Science Research, que coleta as variáveis do ambiente de descanso, já que essas têm influência crucial sobre a qualidade do sono.

\section{Introdução}

O sono é um processo ativo do cérebro humano que tem a função biológica fundamental na consolidação da memória, na visão binocular, na termorregulação, na conservação e restauração da energia [Jansen et al. 2007]. Na sociedade contemporânea é comum relatos de sono insuficiente durante à noite, o que se configura em um cenário preocupante de saúde pública [Liu et al. 2016]. Nesse sentido, para que o sono seja considerado saudável, exige-se uma duração adequada, boa qualidade, regularidade e ausência de pertubações durante sono.

Recomendações da American Academy of Sleep Medicine (AASM) afirmam que um adulto com idade entre 18 a 60 anos necessita em média dormir 7 ou mais horas por noite, regularmente, para inibir resultados adversos à saúde, que incluem o ganho de peso e obesidade, diabetes, hipertensão, doença cardíaca e acidente vascular cerebral, 
depressão e aumento do risco de morte, além do comprometimento da função imunológica [Badr et al. 2015].

Os distúrbios no sono são as causas mais comum de interferência no sono. Um caso bem conhecido é a apnéia obstrutiva do sono, que consiste em um distúrbio fisiológico que provoca repetidas paradas respiratórias durante o período de descanso [Fagundes et al. 2004]. Seu diagnóstico está sujeita a utilização de alguma técnica de monitoramento, que normalmente é a polissonografia realizada em laboratório [de Menezes Duarte et al. 2010]. Contudo, esse método requer múltiplos sensores conectados ao corpo do paciente, e geralmente, o seu deslocamento até uma clínica médica [Bittencourt and Caixeta 2010].

Essa abordagem se mostra um tanto invasiva e notadamente pode afetar o resultado final do processo de triagem, uma vez que o paciente pode sofrer incômodo resultante dos fios dos sensores, além de não se encontrar em seu ambiente normal de sono. Com base na análise das variáveis (luminosidade, ruído, movimento, distância, temperatura e umidade) pretende-se prover informações mais acuradas sobre o descanso dos pacientes sob escrutínio in situ.

Desenvolvido sob a ótica do Design Science Research (DSR), o artefato possui como principal diferencial a avaliação da qualidade do sono in loco, não sendo necessário extirpar o paciente de sua residência, reduzindo custos. Sob essa justificativa, o artigo proposto tem como objetivo apresentar um dispositivo em desenvolvimento para monitoramento não invasivo do ambiente do sono.

\section{Trabalhos relacionados}

As mudanças impostas pelas Tecnologias da Informação e Comunicação (TIC) permitiram o desenvolvimento de sistemas de monitoramento baseados em IoT (Internet das Coisas) que vem sendo amplamente utilizados em muitos domínios de aplicação. Entre os projetos existentes para o monitoramento do sono, pode-se mencionar, o sistema de monitoramento baseado no sensor do Kinect para coletar dados do movimento, postura e do ambiente e a partir desses dados extrair informações gerais sobre o sono [Lee et al. 2015]. Sistemas de reconhecimento contínuo do estado do sono, incluindo os movimento e as saídas do usuário da cama e o monitoramento da respiração do usuário já foram descritos na literatura [Lobato et al. 2015, Lin et al. 2016, Lobato et al. 2017].

Um sistema vestível (colar cervical) de monitoramento do sono não invasivo capaz de monitorar e visualizar em tempo real os sinais fisiológicos foi projetado por [Rofouei et al. 2011]. [Liu et al. 2013] projetou um lençol não invasivo à pressão para o monitoramento da postura do sono. Os mapas de pressão de alta resolução gerados podem ser utilizados para o monitoramento do sono. Já o [Nam et al. 2016] desenvolveu um sistema de monitoramento para quantificar a qualidade do sono.

\section{Arquitetura e funcionalidades do sistema}

O modelo do sistema está dividido em três módulos principais e interdependentes. $\mathrm{O}$ primeiro comporta os sensores utilizados para monitoramento e aquisição das variáveis do ambiente de sono em conjunto com o Kit de desenvolvimento integrado Nvidia JETSON TK ${ }^{1}$. Toda a informação é armazenada em um banco de dados local desenvolvido em

\footnotetext{
${ }^{1}$ https:/www.nvidia.com.br/
} 
SQlite $^{2}$. A implementação dos sensores utilizou a linguagem de programação C++.

Com relação à utilização dos sensores, podemos destacar a importância das variáveis coletadas, como por exemplo, a temperatura ambiente que exerce influência significativa sobre o sono. Estudos revelam que baixas temperaturas prejudicam o sono em humanos, enquanto que altas temperaturas tendem a promover o sono [Jhaveri et al. 2007]. Outro parâmetro que pode afetar a transferência de calor e, consequentemente, a qualidade do sono, é a umidade. Como apontado por [Manzar et al. 2012], várias hipóteses foram propostas para explicar a regulação da estrutura do sono. Para observar essa variações, optou-se pela utilização do sensor de temperatura e umidade DHT22.

É importante também medir o nível de ruído presente no ambiente. Um consenso em relação ao ruído é que esse tem efeitos nocivos para a estrutura do sono, especialmente em termos de fragmentação do sono [Saremi et al. 2008]. O sensor responsável pela captação de ruído no dispositivo em questão é o KY-038. Além disso, a luminosidade também é considerada outro fator que deve ser medido, uma vez que a luz artificial brilhante suprime a secreção noturna da melatonina, o "hormônio do sono" [West et al. 2010]. A aquisição dessa informação está a cargo do sensor de luminosidade BH1750FVI.

No segundo módulo, o sistema provê um servidor que executa uma página web responsiva, contendo questionários/escalas auto-aplicados relevantes a serem usados para realizar uma anamnese complementar do sono, além de ajudar a manter um diário do sono. A interface do sistema bem como os questionários foram implementados utilizando a linguagem de programação Python e a biblioteca Flask ${ }^{3}$.

O terceiro módulo, diz respeito a análise e relatório, cujo objetivo é fornecer informações, acuradas, relativas ao período de monitoramento para auxiliar a equipe técnica no processo de triagem e reduzir os custos associados ao exame. O resumo da arquitetura é apresentado na Figura 1(a), sendo o módulo (1) aquisição dos dados do ambiente, que realiza a coleta dos dados por meio dos sensores, (2) diário do sono, que visa manter informações relevantes sobre o paciente e (3) análise e relatório, que trata da análise dos dados e gera os relatórios para equipe técnica.

A instalação do dispositivo é de fácil realização, como já mencionado, o objetivo é realizar um monitoramento in loco sem a necessidade de deslocamento do paciente até uma clínica. A avaliação é feita na residência do paciente, na qual será observado o que acontece no ambiente de sono enquanto esse dorme. A Figura 1(b) exemplifica um cenário com o dispositivo.

O dispositivo ativa o monitoramento do ambiente por meio de gatilhos. Um exemplo de gatilho seria a detecção de presença de uma pessoa no ambiente. Essa funcionalidade é implementada pelo sensor de movimento PIR DYP-MR003. No entanto, ainda não é suficiente para dizer se o paciente está na cama. Nesse caso, utilizamos o sensor ultrassônico HC-SR04 para medir pequenas distâncias e assim detectar a presença do paciente sobre a cama durante o período de monitoramento.

\footnotetext{
${ }^{2}$ https://www.sqlite.org/

${ }^{3}$ http://flask.pocoo.org/
} 


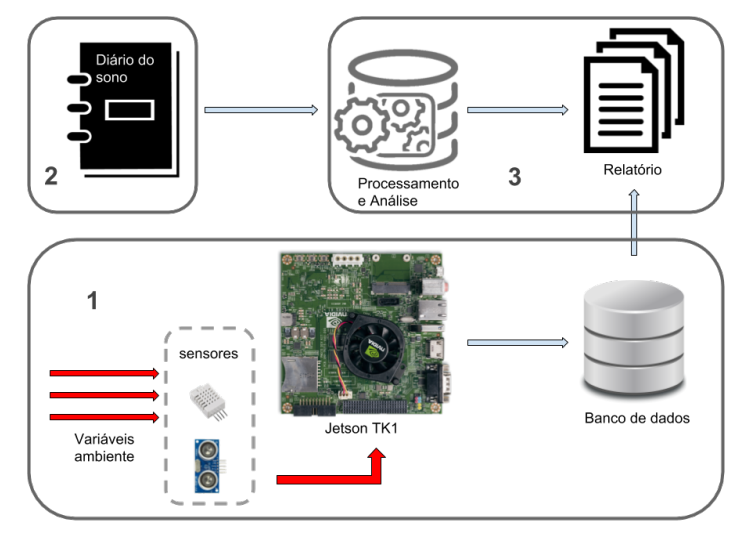

(a) Arquitetura da solução.

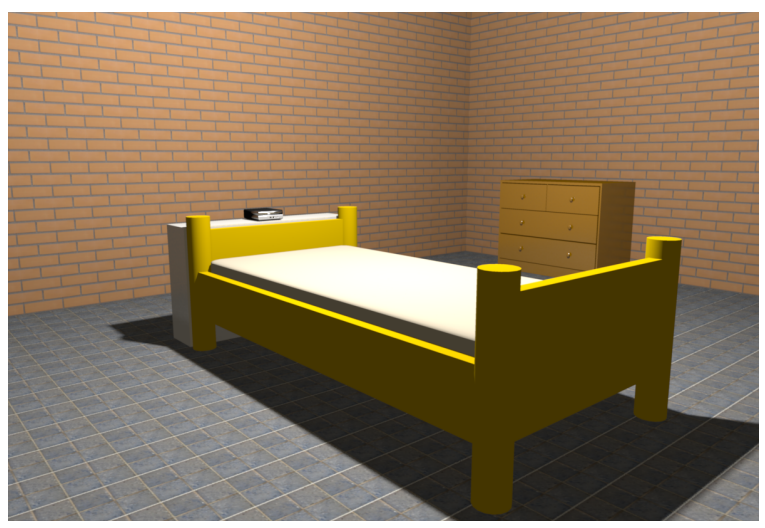

(b) Instalação do dispositivo.

Figura 1. Sistema Proposto

Outra característica do dispositivo é trabalhar com eventos. Uma variação brusca de temperatura ou um ruído de alta intensidade seria considerado um evento importante para análise do sono. Porém, para caracterizar bem um evento, faz-se necessário que heurísticas estejam bem definidas.

Esses eventos detectados devem estar em conjunto com as respostas dos questionários no relatório final. Para, por exemplo, durante o período de monitoramento, informar quantas vezes o paciente levantou da cama, a quantidade de movimentos, os níveis de ruído, a temperatura do ambiente e a umidade. Toda essa informação deve ser bem utilizada para compor a complexa análise do sono.

Até o momento o sistema conta com o servidor web funcional que está armazenando os dados dos questionários. Ademais, três dos cinco sensores pretendidos estão implementados, os quais são: KY-038, PIR DYP-MR003 e HC-SR04; esses já estão coletando as variáveis ambiente e armazenando no banco de dados, o que representa um avanço significativo na implementação do primeiro módulo do sistema, e de crucial importância para que o terceiro módulo tenha todas as variáveis necessárias no cruzamento das informações e geração do relatório. A equipe de desenvolvimento também está trabalhando para futuramente desenvolver uma descrição gráfica, em uma organização cronológica, para facilitar a leitura dos dados dos relatórios.

\section{Cenário de uso}

A exposição do dispositivo estará dividida em diferentes etapas para cada participante que estiver interagindo. No primeiro momento o participante deverá conectar-se à rede do dispositivo e realizar o cadastro por meio de um celular ou computador ao acessar o endereço IP e porta do sistema (127.0.0.0:5000). Em seguida, devem ser respondidos os questionários que serão armazenados no banco de dados.

Na sequência deverá ser demonstrada a ativação do sistema de monitoramento por meio dos gatilhos propostos. O código responsável pelo ativamento automático do sistema estará comentado e a inicialização deverá ser feita manualmente para fins de demonstração. Nessa perspectiva, o dispositivo deverá detectar a presença no ambiente e ativar o monitoramento. Para simular o paciente deitado, será utilizado objetos próximos 
ao sensor de distância ultra-sônico, a partir desse instante, a aquisição dos dados serão realizados e armazenados no banco de dados. A idealização é simular eventos descritos anteriormente - altos níveis de ruído, variação brusca de temperatura e diferentes níveis de luminosidade no ambiente.

Como o dispositivo está em desenvolvimento, a exposição se concentrará em verificar os resultados obtidos pelos sensores em funcionamento. $\mathrm{O}$ banco de dados já permite consultas, e por meio delas será exposto e discutidos os principais dados coletados, assim como, utilizados para realizar uma possível detecção de distúrbio no sono. Algumas condições já são bem conhecidas para essa análise, como por exemplo: um ronco alto deve provocar um ruído excessivo; movimentos seguidos indicariam o paciente rolando de um lado para outro na cama; etc.

Um vídeo compondo alguns resultados da implementação atual encontra-se disponível na plataforma de vídeos Youtube, podendo ser acessado através do link https: //youtu.be/XnXG4zPz7isk.

\section{Agradecimentos}

Os autores gostariam de agradecer à FAPESPA pelas bolsas de iniciação científica concedidas. E também à NVIDIA pela doação do Jetson Development Kit Tk1 utilizadas nos experimentos.

\section{Referências}

Badr, M. S., Belenky, G., Bliwise, D. L., Buxton, O. M., Buysse, D., Dinges, D. F., Gangwisch, J., Grandner, M. A., Kushida, C., Malhotra, R. K., et al. (2015). Recommended amount of sleep for a healthy adult: a joint consensus statement of the american academy of sleep medicine and sleep research society. Journal of Clinical Sleep Medicine, 11(06):591-592.

Bittencourt, L. R. A. and Caixeta, E. C. (2010). Critérios diagnósticos e tratamento dos distúrbios respiratórios do sono: Saos. Jornal Brasileiro de Pneumologia.

de Menezes Duarte, R. L., da Silva, R. Z. M., and da Silveira, F. J. M. (2010). Métodos resumidos no diagnóstico da apnéia do sono. Pulmão RJ, 19(3-4):78-82.

Fagundes, P., Oliveira, D., and Aguirre, L. (2004). Detecção de apnéia durante o sono utilizando modelos identificados. In IFMBE Proc, volume 5, page 1472.

Jansen, J. M., Lopes, A. J., Jansen, U., Capone, D., Maeda, T. Y., Noronha, A., and Magalhães, G. (2007). Medicina da noite: da cronobiologia à prática clínica. SciELOEditora FIOCRUZ.

Jhaveri, K., Trammell, R., and Toth, L. (2007). Effect of environmental temperature on sleep, locomotor activity, core body temperature and immune responses of c57bl/6j mice. Brain, behavior, and immunity, 21(7):975-987.

Lee, J., Hong, M., and Ryu, S. (2015). Sleep monitoring system using kinect sensor. International Journal of Distributed Sensor Networks, 11(10):875371.

Lin, F., Zhuang, Y., Song, C., Wang, A., Li, Y., Gu, C., Li, C., and Xu, W. (2016). SleepSense: A Noncontact and Cost-Effective Sleep Monitoring System. IEEE Transactions on Biomedical Circuits and Systems, 11(1):189-202. 
Liu, J. J., Wenyao Xu, Ming-Chun Huang, Alshurafa, N., Sarrafzadeh, M., Raut, N., and Yadegar, B. (2013). A dense pressure sensitive bedsheet design for unobtrusive sleep posture monitoring. In 2013 IEEE International Conference on Pervasive Computing and Communications (PerCom), pages 207-215. IEEE.

Liu, Y., Wheaton, A. G., Chapman, D. P., Cunningham, T. J., Lu, H., and Croft, J. B. (2016). Prevalence of Healthy Sleep Duration among Adults - United States, 2014. MMWR. Morbidity and Mortality Weekly Report, 65(6):137-141.

Lobato, F., Silva, B., Bem, R., and Miranda, D. (2015). Non-invasive sleep-environment monitoring system. In PETRA '15: Proceedings of the 8th International Conference on PErvasive Technologies Related to Assistive Environments, Corfu, Greece. ACM.

Lobato, F. M. F., de Resende, D. C. O., do Nascimento, R. P., Siqueira, A. L. C., Jacob, A. F. L., and de Santana, Á. L. (2017). Multimodal Low-Invasive System for Sleep Quality Monitoring and Improvement. In Batalla, J. M., Mastorakis, G., Mavromoustakis, C. X., and Pallis, E., editors, Beyond the Internet of Things: Everything Interconnected, pages 223-242. Springer International Publishing, Cham.

Manzar, M. D., Sethi, M., and Hussain, M. E. (2012). Humidity and sleep: a review on thermal aspect. Biological Rhythm Research, 43(4):439-457.

Nam, Y., Kim, Y., and Lee, J. (2016). Sleep monitoring based on a tri-axial accelerometer and a pressure sensor. Sensors, 16(5):1-14.

Rofouei, M., Sinclair, M., Bittner, R., Blank, T., Saw, N., DeJean, G., and Heffron, J. (2011). A Non-invasive Wearable Neck-Cuff System for Real-Time Sleep Monitoring. In 2011 International Conference on Body Sensor Networks, pages 156-161. IEEE.

Saremi, M., Grenèche, J., Bonnefond, A., Rohmer, O., Eschenlauer, A., and Tassi, P. (2008). Effects of nocturnal railway noise on sleep fragmentation in young and middleaged subjects as a function of type of train and sound level. International Journal of Psychophysiology, 70(3):184-191.

West, K. E., Jablonski, M. R., Warfield, B., Cecil, K. S., James, M., Ayers, M. A., Maida, J., Bowen, C., Sliney, D. H., Rollag, M. D., et al. (2010). Blue light from light-emitting diodes (leds) elicits a dose-dependent suppression of melatonin in humans. American Journal of Physiology-Heart and Circulatory Physiology. 